Supplement of Atmos. Chem. Phys. Discuss., 14, 18679-18701, 2014

http://www.atmos-chem-phys-discuss.net/14/18679/2014/

doi:10.5194/acpd-14-18679-2014-supplement

(C) Author(s) 2014. CC Attribution 3.0 License.

(c) (i)

Supplement of

\title{
Methane sulfonic acid enhanced formation of molecular clusters of sulfuric acid and dimethyl amine
}

N. Bork et al.

Correspondence to: N. Bork (nicolai.bork@ helsinki.fi) 


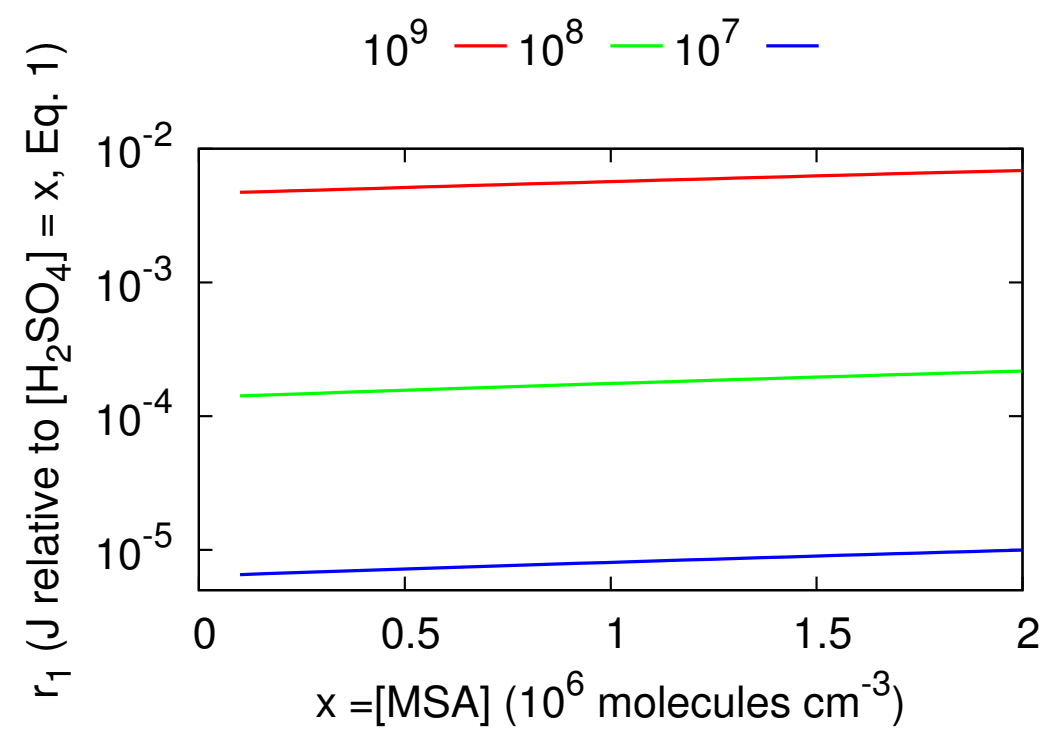

Figure S1: Ratio of MSA-DMA and $\mathrm{H}_{2} \mathrm{SO}_{4}$-DMA based cluster formation rates as defined in Equation 1 in the main article at the indicated DMA concentrations. 


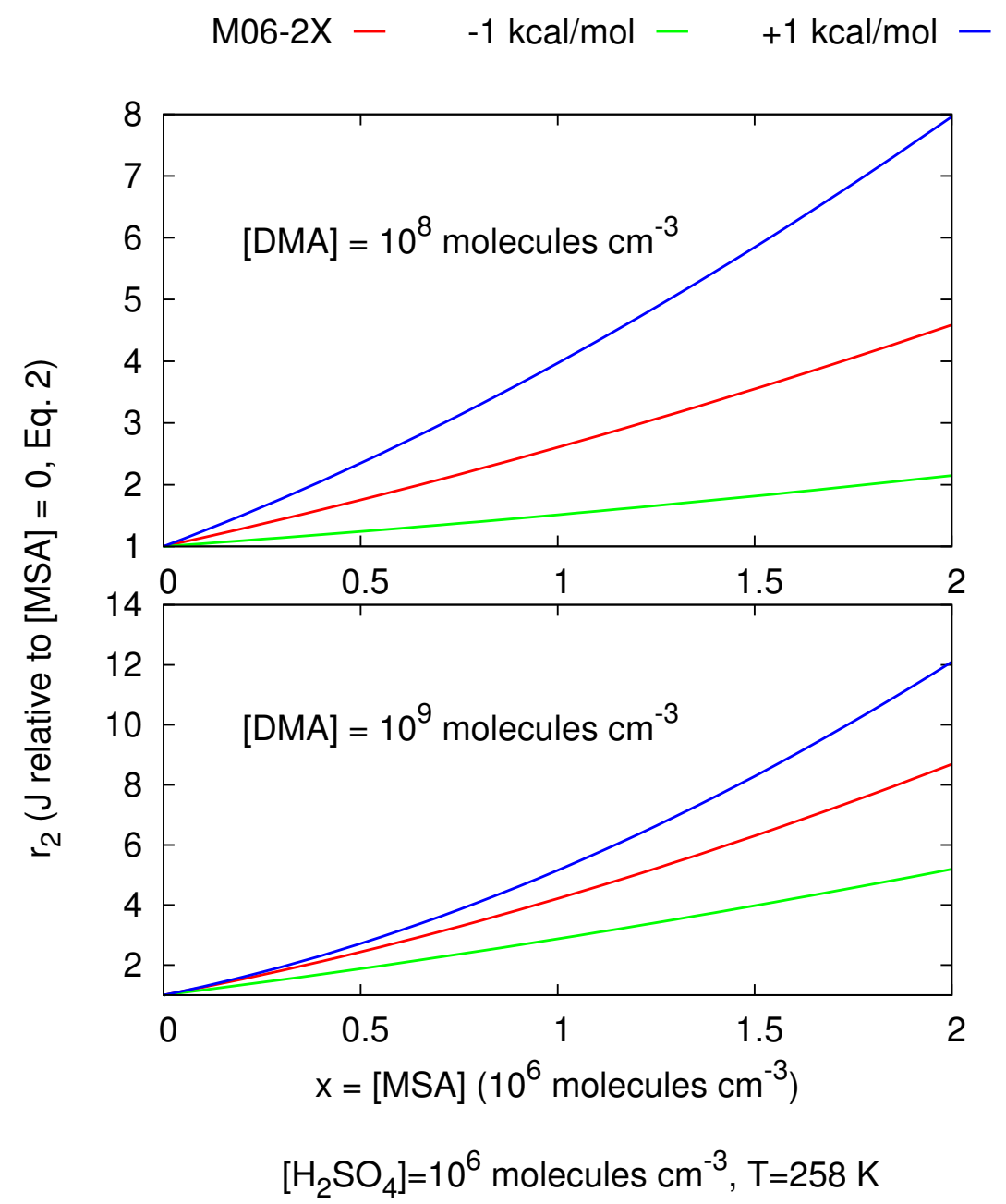

Figure S2: Enhancement factor as defined in Equation 2 in the main article assuming that all clusters are predicted too weakly bound (blue line) or too strongly bound (green line) by $1 \mathrm{kcal} / \mathrm{mol}$, compared to the values presented in the main article (red line) using M06-2X/6-311++G(3df,3pd). 


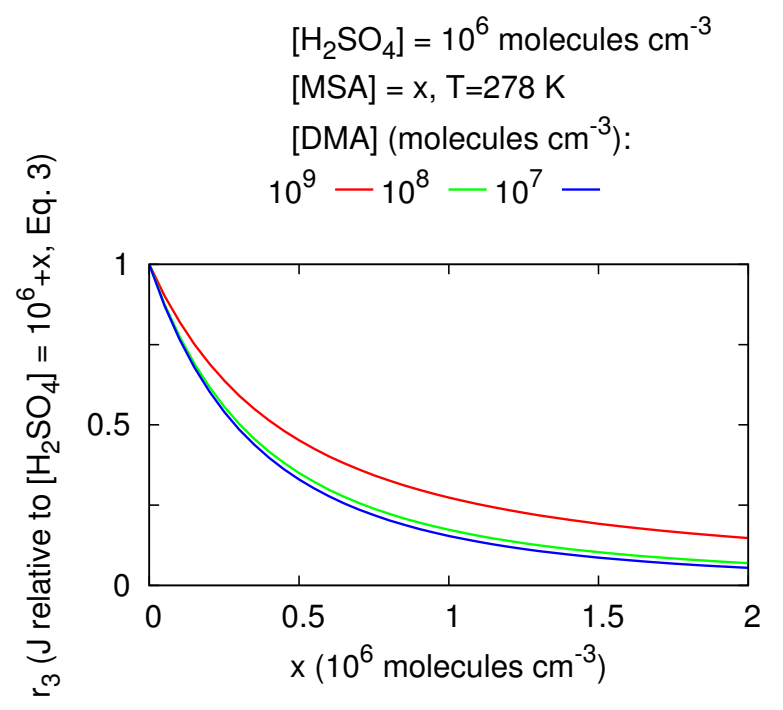

Figure S3: Cluster formation rate of added MSA relative to the same amount of added $\mathrm{H}_{2} \mathrm{SO}_{4}$ as defined in Equation 3 for $\mathrm{T}=278 \mathrm{~K}$.

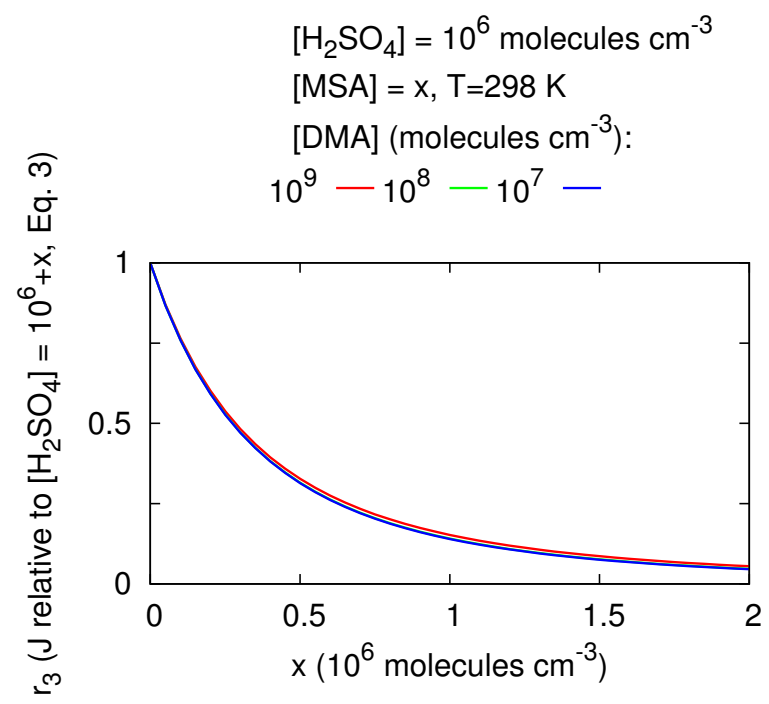

Figure S4: Cluster formation rate of added MSA relative the same amount of added $\mathrm{H}_{2} \mathrm{SO}_{4}$ as defined in Equation 3 for $\mathrm{T}=298 \mathrm{~K}$. 
Table S1: Energy differences between the investigated clusters and corresponding monomers calculated by M06-2X/6-311++G(3df,3pd). SA, MSA and DMA is shorthand for sulfuric acid, methane sulfonic acid and dimethyl amine, respectively. Units are $\mathrm{kcal} / \mathrm{mol}$ and $\mathrm{cal} /(\mathrm{mol} \mathrm{K})$.

\begin{tabular}{|c|c|c|c|}
\hline Species & Binding enthalpy & Binding entropy & Binding Gibbs free energy (298.15 K) \\
\hline \multicolumn{4}{|l|}{ Dimers } \\
\hline$(\mathrm{SA})(\mathrm{DMA})$ & -21.3705 & -33.9220 & -11.2575 \\
\hline$(\mathrm{MSA})(\mathrm{DMA})$ & -18.6747 & -37.7460 & -7.4209 \\
\hline$(\mathrm{SA})_{2}$ & -18.4419 & -33.7590 & -8.3779 \\
\hline$(\mathrm{MSA})_{2}$ & -20.2799 & -37.6040 & -9.0688 \\
\hline$(\mathrm{SA})(\mathrm{MSA})$ & -22.8577 & -40.7860 & -10.6978 \\
\hline$(\mathrm{DMA})_{2}$ & -3.8165 & -27.7800 & 4.4660 \\
\hline \multicolumn{4}{|l|}{ Trimers } \\
\hline$(\mathrm{SA})_{2}(\mathrm{DMA})$ & -53.2260 & -77.3450 & -30.1663 \\
\hline$(\mathrm{SA})(\mathrm{MSA})(\mathrm{DMA})$ & -53.7889 & -83.3270 & -28.9458 \\
\hline$(\mathrm{MSA})_{2}(\mathrm{DMA})$ & -46.1602 & -74.2440 & -24.0248 \\
\hline$(\mathrm{SA})(\mathrm{DMA})_{2}$ & -36.0523 & -73.5250 & -14.1309 \\
\hline$(\mathrm{MSA})(\mathrm{DMA})_{2}$ & -23.6697 & -66.9830 & -3.6985 \\
\hline$(\mathrm{SA})_{3}$ & -36.8141 & -76.7170 & -13.9426 \\
\hline$(\mathrm{MSA})_{3}$ & -32.5652 & -70.8040 & -11.4558 \\
\hline$(\mathrm{SA})_{2}(\mathrm{MSA})$ & -38.4726 & -75.6890 & -15.9080 \\
\hline$(\mathrm{SA})(\mathrm{MSA})_{2}$ & -39.5557 & -77.0310 & -16.5901 \\
\hline \multicolumn{4}{|l|}{ Tetramers } \\
\hline$(\mathrm{SA})_{2}(\mathrm{DMA})_{2}$ & -78.8692 & -118.3660 & -43.5793 \\
\hline$(\mathrm{SA})(\mathrm{MSA})(\mathrm{DMA})_{2}$ & -74.9271 & -117.9480 & -39.7609 \\
\hline$(\mathrm{MSA})_{2}(\mathrm{DMA})_{2}$ & -68.8152 & -107.9090 & -36.6428 \\
\hline$(\mathrm{SA})_{3}(\mathrm{DMA})$ & -77.4635 & -118.7160 & -42.0701 \\
\hline$(\mathrm{SA})_{2}(\mathrm{MSA})(\mathrm{DMA})$ & -78.1400 & -120.3240 & -42.2672 \\
\hline$(\mathrm{SA})(\mathrm{MSA})_{2}(\mathrm{DMA})$ & -73.3132 & -119.8910 & -37.5684 \\
\hline$(\mathrm{MSA})_{3}(\mathrm{DMA})$ & -67.7880 & -119.7310 & -32.0908 \\
\hline \multicolumn{4}{|l|}{ Pentamers } \\
\hline$(\mathrm{MSA})_{3}(\mathrm{DMA})_{2}$ & -91.6823 & -155.3190 & -45.3746 \\
\hline$(\mathrm{SA})(\mathrm{MSA})_{2}(\mathrm{DMA})_{2}$ & -99.8123 & -152.6910 & -54.2884 \\
\hline$(\mathrm{SA})_{2}(\mathrm{MSA})(\mathrm{DMA})_{2}$ & -102.2301 & -156.8580 & -55.4637 \\
\hline$(\mathrm{SA})_{3}(\mathrm{DMA})_{2}$ & -106.4024 & -155.6450 & -59.9987 \\
\hline Hexamer & & & \\
\hline$(\mathrm{MSA})_{1}\left(\mathrm{H}_{2} \mathrm{SO}_{4}\right)_{2}(\mathrm{DMA})_{2}$ & -128.8001 & -197.3050 & -69.9742 \\
\hline
\end{tabular}


Table S2: Geometry of SA. Units are A.

\begin{tabular}{c|ccc} 
Atom & $\mathrm{X}$ & $\mathrm{Y}$ & $\mathrm{Z}$ \\
\hline $\mathrm{S}$ & 0.000063 & 0.000213 & -0.15381100 \\
$\mathrm{O}$ & -0.640599 & 1.069478 & -0.81843000 \\
$\mathrm{O}$ & 0.641011 & -1.067331 & -0.82092200 \\
$\mathrm{O}$ & 1.029406 & 0.661681 & 0.83706000 \\
$\mathrm{H}$ & 1.691454 & 0.00903 & 1.10050100 \\
$\mathrm{O}$ & -1.029838 & -0.663878 & 0.83493300 \\
$\mathrm{H}$ & -1.692302 & -0.012025 & 1.099357
\end{tabular}

Table S3: Geometry of MSA. Units are $\AA$.

\begin{tabular}{c|ccc} 
Atom & $\mathrm{X}$ & $\mathrm{Y}$ & $\mathrm{Z}$ \\
\hline $\mathrm{S}$ & -0.087635 & 0.134824 & 0.06611100 \\
$\mathrm{C}$ & 1.59981 & -0.361314 & -0.02558000 \\
$\mathrm{H}$ & 1.694967 & -1.357427 & 0.39413100 \\
$\mathrm{H}$ & 1.905034 & -0.334378 & -1.06683400 \\
$\mathrm{H}$ & 2.16456 & 0.359484 & 0.55901300 \\
$\mathrm{O}$ & -0.224539 & 1.393873 & -0.57366500 \\
$\mathrm{O}$ & -0.577492 & -0.084946 & 1.38880100 \\
$\mathrm{O}$ & -0.781313 & -0.947124 & -0.87364600 \\
$\mathrm{H}$ & -1.294506 & -1.551402 & -0.322523
\end{tabular}


Table S4: Geometry of DMA. Units are Å.

\begin{tabular}{c|ccc} 
Atom & $\mathrm{X}$ & $\mathrm{Y}$ & $\mathrm{Z}$ \\
\hline $\mathrm{N}$ & -0.000005 & 0.568593 & -0.14905600 \\
$\mathrm{H}$ & -0.000009 & 1.334936 & 0.51053900 \\
$\mathrm{C}$ & 1.204279 & -0.224049 & 0.02025200 \\
$\mathrm{H}$ & 1.261356 & -0.962291 & -0.78040300 \\
$\mathrm{H}$ & 1.240369 & -0.765162 & 0.97608800 \\
$\mathrm{H}$ & 2.082759 & 0.414214 & -0.05075900 \\
$\mathrm{C}$ & -1.204267 & -0.224048 & 0.02024800 \\
$\mathrm{H}$ & -1.261392 & -0.962248 & -0.78044400 \\
$\mathrm{H}$ & -2.082768 & 0.414205 & -0.05068000 \\
$\mathrm{H}$ & -1.240349 & -0.765221 & 0.976058
\end{tabular}

Table S5: Geometry of DMA SA. Units are A.

\begin{tabular}{c|ccc} 
Atom & $\mathrm{X}$ & $\mathrm{Y}$ & $\mathrm{Z}$ \\
\hline $\mathrm{S}$ & -1.267211 & -0.174405 & 0.04944800 \\
$\mathrm{O}$ & -0.553074 & 0.015717 & 1.29327800 \\
$\mathrm{O}$ & -2.472918 & -0.935944 & 0.07942600 \\
$\mathrm{O}$ & -1.690617 & 1.324447 & -0.34847000 \\
$\mathrm{H}$ & -2.476306 & 1.272002 & -0.90388100 \\
$\mathrm{O}$ & -0.334249 & -0.587655 & -1.01995400 \\
$\mathrm{H}$ & 1.040231 & -0.403877 & -0.47126800 \\
$\mathrm{~N}$ & 1.890413 & -0.111827 & 0.15026500 \\
$\mathrm{H}$ & 1.502209 & -0.288162 & 1.08064000 \\
$\mathrm{C}$ & 2.055402 & 1.346068 & -0.01703900 \\
$\mathrm{H}$ & 2.344576 & 1.545194 & -1.04539500 \\
$\mathrm{H}$ & 2.820147 & 1.713356 & 0.66296500 \\
$\mathrm{H}$ & 1.098925 & 1.815548 & 0.19674500 \\
$\mathrm{C}$ & 3.100559 & -0.901202 & -0.11983300 \\
$\mathrm{H}$ & 3.402389 & -0.73476 & -1.15034600 \\
$\mathrm{H}$ & 2.879331 & -1.953878 & 0.02680000 \\
$\mathrm{H}$ & 3.902092 & -0.593866 & 0.54771000
\end{tabular}


Table S6: Geometry of DMA MSA. Units are Å.

\begin{tabular}{c|ccc} 
Atom & $\mathrm{X}$ & $\mathrm{Y}$ & $\mathrm{Z}$ \\
\hline $\mathrm{S}$ & -1.100618 & 0.078767 & -0.04975300 \\
$\mathrm{C}$ & -2.653272 & -0.748458 & 0.12148000 \\
$\mathrm{H}$ & -3.245498 & -0.51027 & -0.75698400 \\
$\mathrm{H}$ & -2.469712 & -1.81597 & 0.18740900 \\
$\mathrm{H}$ & -3.134518 & -0.378092 & 1.02123300 \\
$\mathrm{O}$ & -1.363539 & 1.493065 & -0.11415000 \\
$\mathrm{O}$ & -0.432522 & -0.480243 & -1.21373700 \\
$\mathrm{O}$ & -0.351214 & -0.297244 & 1.19148000 \\
$\mathrm{H}$ & 1.014493 & -0.191819 & 0.89985500 \\
$\mathrm{~N}$ & 2.077886 & -0.031165 & 0.54885200 \\
$\mathrm{H}$ & 2.670933 & 0.04824 & 1.36975500 \\
$\mathrm{C}$ & 2.083583 & 1.245189 & -0.20262300 \\
$\mathrm{H}$ & 1.444817 & 1.115424 & -1.07206400 \\
$\mathrm{H}$ & 3.098831 & 1.495151 & -0.50236700 \\
$\mathrm{H}$ & 1.661827 & 2.023189 & 0.42735000 \\
$\mathrm{C}$ & 2.48538 & -1.189292 & -0.27310100 \\
$\mathrm{H}$ & 1.741561 & -1.299066 & -1.05768400 \\
$\mathrm{H}$ & 2.492374 & -2.079316 & 0.34941000 \\
$\mathrm{H}$ & 3.473637 & -1.018845 & -0.695115
\end{tabular}


Table S7: Geometry of $\mathrm{SA}_{2}$. Units are $\AA$.

\begin{tabular}{c|ccc} 
Atom & $\mathrm{X}$ & $\mathrm{Y}$ & $\mathrm{Z}$ \\
\hline $\mathrm{S}$ & -2.035878 & -0.073295 & 0.11410000 \\
$\mathrm{O}$ & -1.067705 & 0.085136 & 1.15676200 \\
$\mathrm{O}$ & -3.310894 & -0.604497 & 0.39602300 \\
$\mathrm{O}$ & -2.182452 & 1.369579 & -0.49135800 \\
$\mathrm{H}$ & -2.960685 & 1.414409 & -1.06354000 \\
$\mathrm{O}$ & -1.423183 & -0.88934 & -1.03860300 \\
$\mathrm{H}$ & -0.461344 & -0.657678 & -1.15613600 \\
$\mathrm{O}$ & 1.067511 & -0.085828 & -1.15658000 \\
$\mathrm{~S}$ & 2.035801 & 0.073261 & -0.11412700 \\
$\mathrm{O}$ & 1.42284 & 0.889095 & 1.03856700 \\
$\mathrm{O}$ & 3.310397 & 0.605324 & -0.39632300 \\
$\mathrm{O}$ & 2.183503 & -1.36941 & 0.49156900 \\
$\mathrm{H}$ & 0.46111 & 0.657026 & 1.15624300 \\
$\mathrm{H}$ & 2.962028 & -1.413694 & 1.063402
\end{tabular}


Table S8: Geometry of $\mathrm{MSA}_{2}$. Units are $\AA$.

\begin{tabular}{c|ccc} 
Atom & $\mathrm{X}$ & $\mathrm{Y}$ & $\mathrm{Z}$ \\
\hline $\mathrm{S}$ & -1.837512 & 0.088864 & 0.04630000 \\
$\mathrm{O}$ & -1.631683 & 1.490825 & -0.12774400 \\
$\mathrm{O}$ & -1.171603 & -0.530195 & 1.17160800 \\
$\mathrm{O}$ & -1.471288 & -0.689158 & -1.24427400 \\
$\mathrm{~S}$ & 2.011557 & -0.120252 & -0.06790600 \\
$\mathrm{O}$ & 1.477945 & -0.596611 & 1.31252200 \\
$\mathrm{H}$ & 0.484965 & -0.612643 & 1.32817800 \\
$\mathrm{O}$ & 1.119985 & -0.60661 & -1.09926100 \\
$\mathrm{O}$ & 3.38258 & -0.469435 & -0.14712500 \\
$\mathrm{H}$ & -0.472445 & -0.723646 & -1.31458100 \\
$\mathrm{C}$ & -3.550707 & -0.269048 & 0.14202500 \\
$\mathrm{H}$ & -3.672895 & -1.343574 & 0.22948000 \\
$\mathrm{H}$ & -3.921904 & 0.24191 & 1.02576400 \\
$\mathrm{H}$ & -4.02311 & 0.116639 & -0.75591000 \\
$\mathrm{C}$ & 1.839751 & 1.628769 & 0.00431200 \\
$\mathrm{H}$ & 2.44072 & 1.98612 & 0.83455000 \\
$\mathrm{H}$ & 2.211322 & 2.018194 & -0.93957200 \\
$\mathrm{H}$ & 0.786865 & 1.870352 & 0.133941
\end{tabular}


Table S9: Geometry of SA MSA. Units are A.

\begin{tabular}{c|ccc} 
Atom & $\mathrm{X}$ & $\mathrm{Y}$ & $\mathrm{Z}$ \\
\hline $\mathrm{S}$ & -1.725635 & 0.001889 & -0.06574300 \\
$\mathrm{C}$ & -3.470936 & 0.002544 & -0.18999500 \\
$\mathrm{O}$ & -1.196641 & -1.204753 & -0.64397200 \\
$\mathrm{O}$ & -1.200268 & 1.237458 & -0.58241100 \\
$\mathrm{O}$ & -1.563526 & -0.037073 & 1.47832300 \\
$\mathrm{~S}$ & 1.930346 & -0.001246 & 0.08937900 \\
$\mathrm{O}$ & 1.5547 & 1.236764 & -0.77108300 \\
$\mathrm{H}$ & 0.584065 & 1.388218 & -0.76120900 \\
$\mathrm{O}$ & 1.06546 & -0.020837 & 1.23930600 \\
$\mathrm{O}$ & 3.325213 & -0.001707 & 0.25726700 \\
$\mathrm{O}$ & 1.559525 & -1.212847 & -0.80988100 \\
$\mathrm{H}$ & 0.588231 & -1.360162 & -0.81455900 \\
$\mathrm{H}$ & -0.589652 & -0.038628 & 1.66696000 \\
$\mathrm{H}$ & -3.842552 & -0.908648 & 0.26786000 \\
$\mathrm{H}$ & -3.700831 & 0.029374 & -1.25140900 \\
$\mathrm{H}$ & -3.844727 & 0.888249 & 0.313766
\end{tabular}


Table S10: Geometry of DMA SA $\mathrm{S}_{2}$. Units are $\AA$.

\begin{tabular}{c|ccc} 
Atom & $\mathrm{X}$ & $\mathrm{Y}$ & $\mathrm{Z}$ \\
\hline $\mathrm{S}$ & 2.231577 & 0.028976 & 0.02757100 \\
$\mathrm{O}$ & 1.318603 & 1.06914 & -0.38957900 \\
$\mathrm{O}$ & 3.59381 & 0.349805 & 0.18880300 \\
$\mathrm{O}$ & 1.696466 & -0.563379 & 1.35199500 \\
$\mathrm{H}$ & 0.693365 & -0.629809 & 1.32677100 \\
$\mathrm{O}$ & 2.127857 & -1.136989 & -0.98057900 \\
$\mathrm{H}$ & 1.186944 & -1.45851 & -1.04312100 \\
$\mathrm{~S}$ & -1.416599 & -1.244316 & -0.10513900 \\
$\mathrm{O}$ & -0.878388 & -0.615331 & 1.10468700 \\
$\mathrm{O}$ & -0.386891 & -1.915618 & -0.87182900 \\
$\mathrm{O}$ & -2.410617 & -2.375884 & 0.37282200 \\
$\mathrm{H}$ & -1.906976 & -3.145181 & 0.66658800 \\
$\mathrm{O}$ & -2.264284 & -0.343175 & -0.84641300 \\
$\mathrm{H}$ & -1.768465 & 1.270039 & -0.41880200 \\
$\mathrm{~N}$ & -1.180882 & 2.052867 & -0.06939600 \\
$\mathrm{H}$ & -0.240759 & 1.621913 & 0.02507600 \\
$\mathrm{C}$ & -1.673905 & 2.497327 & 1.25004700 \\
$\mathrm{H}$ & -2.663744 & 2.929444 & 1.13164600 \\
$\mathrm{H}$ & -0.989994 & 3.241104 & 1.64951700 \\
$\mathrm{H}$ & -1.714646 & 1.631303 & 1.90273600 \\
$\mathrm{C}$ & -1.086714 & 3.124486 & -1.08103700 \\
$\mathrm{H}$ & -2.072059 & 3.551852 & -1.24455500 \\
$\mathrm{H}$ & -0.703138 & 2.696143 & -2.00163900 \\
$\mathrm{H}$ & -0.402733 & 3.887646 & -0.720668
\end{tabular}


Table S11: Geometry of DMA MSA 2 . Units are $\AA$.

\begin{tabular}{c|ccc} 
Atom & $\mathrm{X}$ & $\mathrm{Y}$ & $\mathrm{Z}$ \\
\hline $\mathrm{S}$ & 2.303408 & -0.283323 & -0.09729600 \\
$\mathrm{C}$ & 2.207511 & -0.964188 & 1.52249100 \\
$\mathrm{H}$ & 1.157055 & -1.044764 & 1.78901800 \\
$\mathrm{H}$ & 2.69331 & -1.934869 & 1.49944400 \\
$\mathrm{H}$ & 2.7403 & -0.285426 & 2.18245200 \\
$\mathrm{O}$ & 3.665634 & -0.242987 & -0.50525400 \\
$\mathrm{O}$ & 1.574005 & 0.971625 & -0.08667200 \\
$\mathrm{O}$ & 1.551039 & -1.296749 & -0.97003900 \\
$\mathrm{H}$ & 0.528271 & -1.124033 & -1.04174000 \\
$\mathrm{~S}$ & -1.774458 & -0.905025 & -0.02497200 \\
$\mathrm{C}$ & -2.596528 & -2.465886 & -0.04923500 \\
$\mathrm{H}$ & -3.14411 & -2.543316 & -0.98318800 \\
$\mathrm{H}$ & -1.841883 & -3.243023 & 0.02525900 \\
$\mathrm{H}$ & -3.270856 & -2.498116 & 0.80095600 \\
$\mathrm{O}$ & -0.895 & -0.881032 & -1.21425400 \\
$\mathrm{O}$ & -1.005833 & -0.821864 & 1.20289500 \\
$\mathrm{O}$ & -2.808946 & 0.1177 & -0.13595300 \\
$\mathrm{H}$ & -1.743793 & 1.539129 & -0.23845500 \\
$\mathrm{~N}$ & -0.871976 & 2.07249 & -0.05341500 \\
$\mathrm{H}$ & -0.085626 & 1.400711 & -0.16035600 \\
$\mathrm{C}$ & -0.870775 & 2.50385 & 1.36034600 \\
$\mathrm{H}$ & -1.655347 & 3.240296 & 1.51093800 \\
$\mathrm{H}$ & 0.101582 & 2.932105 & 1.58795300 \\
$\mathrm{H}$ & -1.04893 & 1.623316 & 1.97128600 \\
$\mathrm{C}$ & -0.670588 & 3.161871 & -1.02660800 \\
$\mathrm{H}$ & -1.451488 & 3.906792 & -0.90114100 \\
$\mathrm{H}$ & -0.7084 & 2.744128 & -2.02784800 \\
$\mathrm{H}$ & 0.305652 & 3.605795 & -0.852127
\end{tabular}


Table S12: Geometry of DMA SA MSA. Units are A.

\begin{tabular}{c|ccc} 
Atom & $\mathrm{X}$ & $\mathrm{Y}$ & $\mathrm{Z}$ \\
\hline $\mathrm{S}$ & 2.326966 & 0.035878 & -0.07242900 \\
$\mathrm{O}$ & 3.73458 & 0.064753 & -0.15192200 \\
$\mathrm{O}$ & 1.591831 & 1.27003 & -0.16700400 \\
$\mathrm{O}$ & 1.78841 & -0.919672 & -1.16951000 \\
$\mathrm{H}$ & 0.793895 & -0.822561 & -1.23928200 \\
$\mathrm{O}$ & 1.937554 & -0.666581 & 1.25148300 \\
$\mathrm{H}$ & 0.95635 & -0.870321 & 1.28064100 \\
$\mathrm{~S}$ & -1.51439 & -1.083408 & 0.07352100 \\
$\mathrm{C}$ & -1.969655 & -2.731984 & -0.35017400 \\
$\mathrm{H}$ & -1.062302 & -3.288409 & -0.56599200 \\
$\mathrm{H}$ & -2.489402 & -3.157795 & 0.50273600 \\
$\mathrm{H}$ & -2.618501 & -2.689565 & -1.21931300 \\
$\mathrm{O}$ & -0.617369 & -1.177922 & 1.22385100 \\
$\mathrm{O}$ & -2.716969 & -0.327277 & 0.32806800 \\
$\mathrm{O}$ & -0.789004 & -0.542597 & -1.10628500 \\
$\mathrm{H}$ & -0.762425 & 1.169544 & -0.56391600 \\
$\mathrm{~N}$ & -0.919232 & 2.140137 & -0.22517400 \\
$\mathrm{H}$ & -0.084481 & 2.639057 & -0.53315200 \\
$\mathrm{C}$ & -0.956025 & 2.143364 & 1.26112100 \\
$\mathrm{H}$ & -1.800702 & 1.538155 & 1.57408600 \\
$\mathrm{H}$ & -1.064785 & 3.170439 & 1.59916800 \\
$\mathrm{H}$ & -0.026218 & 1.715341 & 1.62050000 \\
$\mathrm{C}$ & -2.156775 & 2.662175 & -0.84438700 \\
$\mathrm{H}$ & -2.969618 & 2.007779 & -0.54260700 \\
$\mathrm{H}$ & -2.046739 & 2.643109 & -1.92406800 \\
$\mathrm{H}$ & -2.3292 & 3.677529 & -0.498847
\end{tabular}


Table S13: Geometry of $\mathrm{DMA}_{2} \mathrm{SA}_{2}$. Units are $\AA$.

\begin{tabular}{c|ccc} 
Atom & $\mathrm{X}$ & $\mathrm{Y}$ & $\mathrm{Z}$ \\
\hline $\mathrm{N}$ & -2.777147 & -0.578147 & 0.06846700 \\
$\mathrm{H}$ & -2.11094 & 0.173922 & -0.21958900 \\
$\mathrm{C}$ & -3.109701 & -0.448095 & 1.50233100 \\
$\mathrm{H}$ & -3.871639 & -1.181206 & 1.75420000 \\
$\mathrm{H}$ & -3.4716 & 0.558212 & 1.69247800 \\
$\mathrm{H}$ & -2.208253 & -0.634585 & 2.07703700 \\
$\mathrm{C}$ & -3.94475 & -0.509746 & -0.83204300 \\
$\mathrm{H}$ & -4.620043 & -1.330528 & -0.60774800 \\
$\mathrm{H}$ & -3.59614 & -0.581689 & -1.85762800 \\
$\mathrm{H}$ & -4.448812 & 0.441208 & -0.68309100 \\
$\mathrm{~S}$ & -0.277839 & 2.096862 & -0.17547300 \\
$\mathrm{O}$ & 1.158498 & 1.87648 & -0.28002500 \\
$\mathrm{O}$ & -0.720684 & 3.440873 & -0.27685100 \\
$\mathrm{O}$ & -0.997494 & 1.152325 & -1.06886600 \\
$\mathrm{H}$ & -0.067414 & -0.167343 & -1.38556300 \\
$\mathrm{O}$ & -0.68917 & 1.615957 & 1.28229700 \\
$\mathrm{H}$ & -0.353709 & 0.69595 & 1.36796200 \\
$\mathrm{~S}$ & 0.258751 & -1.86063 & -0.10688000 \\
$\mathrm{O}$ & 0.497598 & -0.988746 & -1.40449000 \\
$\mathrm{O}$ & 0.129135 & -0.895567 & 0.99203500 \\
$\mathrm{O}$ & -0.972285 & -2.604374 & -0.30326000 \\
$\mathrm{H}$ & -2.242127 & -1.463351 & -0.08338200 \\
$\mathrm{O}$ & 1.461348 & -2.648273 & -0.01189700 \\
$\mathrm{H}$ & 2.604809 & -1.12515 & -0.03788500 \\
$\mathrm{~N}$ & 2.87478 & -0.138727 & 0.10021900 \\
$\mathrm{H}$ & 2.025377 & 0.445532 & -0.07815500 \\
$\mathrm{C}$ & 3.896047 & 0.273876 & -0.88137400 \\
$\mathrm{H}$ & 4.812195 & -0.284768 & -0.71058300 \\
$\mathrm{H}$ & 4.0734 & 1.33909 & -0.76245500 \\
$\mathrm{H}$ & 3.512143 & 0.075981 & -1.87743000 \\
$\mathrm{C}$ & 3.260892 & 0.070819 & 1.50996400 \\
$\mathrm{H}$ & 4.175497 & -0.476733 & 1.72073600 \\
$\mathrm{H}$ & 2.449239 & -0.291891 & 2.13339100 \\
$\mathrm{H}$ & 3.409485 & 1.135235 & 1.669736
\end{tabular}


Table S14: Geometry of $\mathrm{DMA}_{2} \mathrm{MSA}_{2}$. Units are $\AA$.

\begin{tabular}{c|ccc} 
Atom & $\mathrm{X}$ & $\mathrm{Y}$ & $\mathrm{Z}$ \\
\hline $\mathrm{H}$ & -0.607653 & -1.398838 & 0.26380200 \\
$\mathrm{~S}$ & 2.64552 & -0.042384 & -0.23190100 \\
$\mathrm{O}$ & 2.426412 & -1.3637 & -0.82519800 \\
$\mathrm{O}$ & 2.641277 & 1.050373 & -1.17787400 \\
$\mathrm{O}$ & 1.707447 & 0.186176 & 0.88271200 \\
$\mathrm{~N}$ & 0.03016 & -2.209236 & 0.12834700 \\
$\mathrm{H}$ & 0.905005 & -1.85775 & -0.32599200 \\
$\mathrm{C}$ & -0.608081 & -3.227103 & -0.72799300 \\
$\mathrm{H}$ & -1.527511 & -3.56186 & -0.25626100 \\
$\mathrm{H}$ & 0.082255 & -4.057607 & -0.85308100 \\
$\mathrm{H}$ & -0.849959 & -2.775906 & -1.68349100 \\
$\mathrm{C}$ & 0.391941 & -2.696652 & 1.47314500 \\
$\mathrm{H}$ & -0.509842 & -3.021892 & 1.98533900 \\
$\mathrm{H}$ & 0.857148 & -1.874577 & 2.00815700 \\
$\mathrm{H}$ & 1.089304 & -3.524542 & 1.37435600 \\
$\mathrm{H}$ & 0.607891 & 1.398971 & 0.26390400 \\
$\mathrm{~S}$ & -2.645497 & 0.042304 & -0.23196200 \\
$\mathrm{O}$ & -1.707521 & -0.186117 & 0.88275500 \\
$\mathrm{O}$ & -2.426115 & 1.363407 & -0.82562700 \\
$\mathrm{O}$ & -2.641417 & -1.050711 & -1.17762800 \\
$\mathrm{~N}$ & -0.030058 & 2.209222 & 0.12811900 \\
$\mathrm{H}$ & -0.904753 & 1.857446 & -0.32627500 \\
$\mathrm{C}$ & 0.608106 & 3.22696 & -0.72843200 \\
$\mathrm{H}$ & 1.527388 & 3.562068 & -0.25665700 \\
$\mathrm{H}$ & -0.082381 & 4.057283 & -0.85388100 \\
$\mathrm{H}$ & 0.850222 & 2.775525 & -1.68375900 \\
$\mathrm{C}$ & -0.392195 & 2.696999 & 1.47268700 \\
$\mathrm{H}$ & 0.509407 & 3.022673 & 1.98492500 \\
$\mathrm{H}$ & -0.857279 & 1.874986 & 2.00790200 \\
$\mathrm{H}$ & -1.089769 & 3.524661 & 1.37349200 \\
$\mathrm{C}$ & 4.261028 & -0.103672 & 0.48822800 \\
$\mathrm{H}$ & 4.973116 & -0.286229 & -0.31081500 \\
$\mathrm{H}$ & 4.454277 & 0.853211 & 0.96331300 \\
$\mathrm{H}$ & 4.283929 & -0.90878 & 1.21605700 \\
$\mathrm{C}$ & -4.26101 & 0.104094 & 0.48808500 \\
$\mathrm{H}$ & -4.973022 & 0.286594 & -0.31104100 \\
$\mathrm{H}$ & -4.454475 & -0.852625 & 0.96340800 \\
$\mathrm{H}$ & -4.283775 & 0.909391 & 1.215706
\end{tabular}


Table S15: Geometry of $\mathrm{DMA}_{2} \mathrm{SA}$ MSA. Units are $\AA$.

\begin{tabular}{c|ccc} 
Atom & $\mathrm{X}$ & $\mathrm{Y}$ & $\mathrm{Z}$ \\
\hline $\mathrm{N}$ & -2.47052 & -0.965765 & 0.39995100 \\
$\mathrm{H}$ & -1.85682 & -1.51988 & -0.23315600 \\
$\mathrm{C}$ & -3.68966 & -0.526641 & -0.30012500 \\
$\mathrm{H}$ & -4.285878 & 0.078508 & 0.37783900 \\
$\mathrm{H}$ & -4.255568 & -1.398083 & -0.61904200 \\
$\mathrm{H}$ & -3.387465 & 0.067501 & -1.15712800 \\
$\mathrm{C}$ & -2.710381 & -1.772746 & 1.61080300 \\
$\mathrm{H}$ & -3.278007 & -1.180899 & 2.32410200 \\
$\mathrm{H}$ & -1.742527 & -2.040158 & 2.02320400 \\
$\mathrm{H}$ & -3.264865 & -2.667117 & 1.33939300 \\
$\mathrm{~S}$ & -0.661743 & 1.926962 & -0.09000600 \\
$\mathrm{O}$ & 0.774789 & 1.981654 & 0.16306300 \\
$\mathrm{O}$ & -1.000415 & 1.090902 & -1.25154400 \\
$\mathrm{H}$ & 0.174085 & -0.029601 & -1.58879800 \\
$\mathrm{O}$ & -1.466617 & 1.57216 & 1.07081500 \\
$\mathrm{~S}$ & 0.575719 & -1.794631 & -0.46481400 \\
$\mathrm{O}$ & 0.893432 & -0.713386 & -1.58626800 \\
$\mathrm{O}$ & 0.282609 & -1.029942 & 0.74500000 \\
$\mathrm{O}$ & -0.590646 & -2.539304 & -0.91149300 \\
$\mathrm{H}$ & -1.924244 & -0.125087 & 0.66606400 \\
$\mathrm{O}$ & 1.817474 & -2.526023 & -0.38108200 \\
$\mathrm{H}$ & 2.706415 & -0.836507 & 0.16024900 \\
$\mathrm{~N}$ & 2.770324 & 0.154296 & 0.43903800 \\
$\mathrm{H}$ & 1.833975 & 0.572375 & 0.26031100 \\
$\mathrm{C}$ & 3.749615 & 0.866038 & -0.40118900 \\
$\mathrm{H}$ & 4.745033 & 0.471573 & -0.21515300 \\
$\mathrm{H}$ & 3.707399 & 1.923288 & -0.15523800 \\
$\mathrm{H}$ & 3.471405 & 0.716626 & -1.44002000 \\
$\mathrm{C}$ & 3.011029 & 0.249413 & 1.89146300 \\
$\mathrm{H}$ & 3.980817 & -0.180324 & 2.12760100 \\
$\mathrm{H}$ & 2.214583 & -0.294427 & 2.38974800 \\
$\mathrm{H}$ & 2.979961 & 1.297869 & 2.17465600 \\
$\mathrm{C}$ & -1.124885 & 3.575535 & -0.53064200 \\
$\mathrm{H}$ & -0.896345 & 4.222599 & 0.31076600 \\
$\mathrm{H}$ & -0.554084 & 3.864124 & -1.40780900 \\
$\mathrm{H}$ & -2.189432 & 3.582533 & -0.743201 \\
& & &
\end{tabular}


Table S16: Geometry of $\mathrm{DMA}_{2} \mathrm{SA}_{3}$. Units are $\AA$.

\begin{tabular}{c|ccc} 
Atom & $\mathrm{X}$ & $\mathrm{Y}$ & $\mathrm{Z}$ \\
\hline $\mathrm{S}$ & -3.820794 & -0.614876 & -0.49062900 \\
$\mathrm{O}$ & -5.119344 & -0.478945 & -1.02136300 \\
$\mathrm{O}$ & -3.337108 & 0.355554 & 0.45668800 \\
$\mathrm{O}$ & -3.704431 & -2.016768 & 0.14977300 \\
$\mathrm{H}$ & -2.801157 & -2.119583 & 0.55027700 \\
$\mathrm{O}$ & -2.818651 & -0.654698 & -1.67801900 \\
$\mathrm{H}$ & -1.880093 & -0.606296 & -1.34646200 \\
$\mathrm{~N}$ & 3.743456 & -1.49119 & -0.15913500 \\
$\mathrm{H}$ & 3.394317 & -0.679534 & 0.40077200 \\
$\mathrm{C}$ & 4.824819 & -1.039116 & -1.06309400 \\
$\mathrm{H}$ & 5.134563 & -1.872844 & -1.68728800 \\
$\mathrm{H}$ & 5.65845 & -0.696594 & -0.45687900 \\
$\mathrm{H}$ & 4.453127 & -0.217007 & -1.66648000 \\
$\mathrm{C}$ & 4.121245 & -2.602119 & 0.73975300 \\
$\mathrm{H}$ & 4.446017 & -3.448596 & 0.14095200 \\
$\mathrm{H}$ & 3.250648 & -2.87158 & 1.32839800 \\
$\mathrm{H}$ & 4.932884 & -2.270363 & 1.38082400 \\
$\mathrm{~S}$ & 2.404369 & 1.54007 & -0.32183100 \\
$\mathrm{O}$ & 1.290391 & 2.457293 & -0.19709700 \\
$\mathrm{O}$ & 3.653564 & 2.073516 & -0.74688300 \\
$\mathrm{O}$ & 2.526232 & 0.707198 & 0.90385300 \\
$\mathrm{H}$ & 1.133572 & 0.13915 & 1.49783000 \\
$\mathrm{O}$ & 2.012522 & 0.502335 & -1.46222800 \\
$\mathrm{H}$ & 1.096524 & 0.171436 & -1.31136800 \\
$\mathrm{~S}$ & -0.073186 & -1.288943 & 0.52382100 \\
$\mathrm{O}$ & 0.237117 & -0.273177 & 1.68010800 \\
$\mathrm{O}$ & -0.40681 & -0.435161 & -0.64584700 \\
$\mathrm{O}$ & 1.128749 & -2.048148 & 0.28388600 \\
$\mathrm{H}$ & 2.901031 & -1.755296 & -0.67853300 \\
$\mathrm{O}$ & -1.208254 & -2.04313 & 0.98249300 \\
$\mathrm{H}$ & -1.678825 & 1.18118 & 0.55517200 \\
$\mathrm{~N}$ & -1.270675 & 2.120445 & 0.62502000 \\
$\mathrm{H}$ & -0.257903 & 2.061089 & 0.40572800 \\
$\mathrm{C}$ & -1.468876 & 2.614233 & 2.00170500 \\
$\mathrm{H}$ & -2.535441 & 2.648274 & 2.20556600 \\
$\mathrm{H}$ & -1.031907 & 3.60522 & 2.08725700 \\
$\mathrm{H}$ & -0.981862 & 1.925869 & 2.68525700 \\
$\mathrm{H}$ & -1.907183 & 2.959974 & -0.41152300 \\
& -2.974556 & 3.003463 & -0.21740000 \\
$\mathrm{H}$ & -1.725762 & 2.502437 & -1.37970500 \\
-1.461156 & 3.949999 & -0.380852
\end{tabular}


Table S17: Geometry of $\mathrm{DMA}_{2} \mathrm{MSA}_{3}$. Units are $\AA$.

\begin{tabular}{c|ccc} 
Atom & $\mathrm{X}$ & $\mathrm{Y}$ & $\mathrm{Z}$ \\
\hline $\mathrm{S}$ & 3.833352 & -0.637394 & -0.00336900 \\
$\mathrm{C}$ & 5.55806 & -0.976843 & 0.01836500 \\
$\mathrm{H}$ & 5.706442 & -1.784056 & 0.72959800 \\
$\mathrm{H}$ & 5.85987 & -1.276962 & -0.97978400 \\
$\mathrm{H}$ & 6.078449 & -0.079724 & 0.33787200 \\
$\mathrm{O}$ & 3.417116 & -0.173021 & 1.28940700 \\
$\mathrm{O}$ & 3.133895 & -1.780334 & -0.52741500 \\
$\mathrm{O}$ & 3.796567 & 0.51938 & -1.02493600 \\
$\mathrm{H}$ & 2.805039 & 0.80864 & -1.14531600 \\
$\mathrm{H}$ & -2.436441 & 0.983108 & -0.18989300 \\
$\mathrm{~S}$ & 0.694498 & 1.942079 & -0.17417400 \\
$\mathrm{O}$ & -0.138896 & 2.925328 & -0.84813500 \\
$\mathrm{O}$ & -0.019689 & 1.193082 & 0.84515600 \\
$\mathrm{O}$ & 1.36457 & 1.030611 & -1.13478100 \\
$\mathrm{~N}$ & -2.679492 & 1.989406 & -0.05151000 \\
$\mathrm{H}$ & -1.843595 & 2.505627 & -0.37355900 \\
$\mathrm{C}$ & -2.919005 & 2.254361 & 1.38334800 \\
$\mathrm{H}$ & -3.741684 & 1.624709 & 1.70746700 \\
$\mathrm{H}$ & -3.158386 & 3.307463 & 1.50981100 \\
$\mathrm{H}$ & -2.013683 & 2.001419 & 1.92487400 \\
$\mathrm{C}$ & -3.849207 & 2.275795 & -0.90336600 \\
$\mathrm{H}$ & -4.664158 & 1.637016 & -0.57420600 \\
$\mathrm{H}$ & -3.593 & 2.049925 & -1.93407500 \\
$\mathrm{H}$ & -4.11901 & 3.324154 & -0.80601400 \\
$\mathrm{H}$ & 0.957348 & -0.879998 & -0.31470200 \\
$\mathrm{H}$ & -3.228845 & -1.403595 & 0.07805900 \\
$\mathrm{H}$ & -4.687193 & -1.723513 & -1.74090200 \\
$\mathrm{O}$ & -2.280319 & -0.626985 & -0.76294300 \\
$\mathrm{O}$ & -2.518867 & -2.325838 & 0.94042700 \\
$\mathrm{O}$ & -4.174091 & -0.520806 & 0.73340500 \\
$\mathrm{~N}$ & 0.257864 & -1.521868 & 0.06824800 \\
$\mathrm{H}$ & -0.641641 & -1.01845 & 0.00784100 \\
$\mathrm{C}$ & 0.581962 & -1.827318 & 1.47965200 \\
$\mathrm{H}$ & 1.529236 & -2.357702 & 1.50119700 \\
$\mathrm{H}$ & -0.229104 & -2.428467 & 1.87899000 \\
$\mathrm{H}$ & 0.669424 & -0.888002 & 2.01430200 \\
$\mathrm{C}$ & 0.162044 & -2.725625 & -0.78214100 \\
$\mathrm{H}$ & 1.12611 & -3.223809 & -0.78463500 \\
$\mathrm{H}$ & -0.10693 & -2.414953 & -1.78720400 \\
$\mathrm{H}$ & -0.616147 & -3.359491 & -0.36700100 \\
$\mathrm{H}$ & 1.599144 & 2.822555 & 0.62531000 \\
$\mathrm{H}$ & 2.631878 & 2.09717 & 1.13094200 \\
$\mathrm{H}$ & -3.5964 & 3.368761 & -0.13335400 \\
$\mathrm{H}$ & -2.026204 & -1.610088
\end{tabular}


Table S18: Geometry of $\mathrm{DMA}_{2} \mathrm{SA}_{2} \mathrm{MSA}$. Units are $\AA$.

\begin{tabular}{c|ccc} 
Atom & $\mathrm{X}$ & $\mathrm{Y}$ & $\mathrm{Z}$ \\
\hline $\mathrm{S}$ & 3.969449 & -0.435674 & 0.07006900 \\
$\mathrm{C}$ & 3.754733 & -1.424963 & 1.51286800 \\
$\mathrm{H}$ & 4.013181 & -0.80228 & 2.36454900 \\
$\mathrm{H}$ & 2.713245 & -1.733989 & 1.55739100 \\
$\mathrm{H}$ & 4.424155 & -2.276045 & 1.43836900 \\
$\mathrm{O}$ & 5.339819 & -0.083926 & -0.07880100 \\
$\mathrm{O}$ & 2.996309 & 0.627099 & 0.12821500 \\
$\mathrm{O}$ & 3.603216 & -1.402198 & -1.08294700 \\
$\mathrm{H}$ & 2.646192 & -1.701976 & -0.99022700 \\
$\mathrm{~N}$ & 0.853806 & 2.290418 & 0.00221000 \\
$\mathrm{H}$ & 1.081774 & 1.290806 & 0.04234200 \\
$\mathrm{C}$ & 1.399866 & 2.964692 & 1.19668100 \\
$\mathrm{H}$ & 1.141423 & 4.018977 & 1.14993100 \\
$\mathrm{H}$ & 2.476217 & 2.827245 & 1.20734000 \\
$\mathrm{H}$ & 0.954893 & 2.515456 & 2.07965900 \\
$\mathrm{C}$ & 1.387507 & 2.812944 & -1.27469500 \\
$\mathrm{H}$ & 1.095108 & 3.854714 & -1.37242300 \\
$\mathrm{H}$ & 0.965126 & 2.22306 & -2.08217800 \\
$\mathrm{H}$ & 2.467584 & 2.707161 & -1.26363600 \\
$\mathrm{~S}$ & 0.085829 & -1.351423 & -0.34372500 \\
$\mathrm{O}$ & -1.18565 & -2.048058 & -0.33551000 \\
$\mathrm{O}$ & 1.22798 & -2.217573 & -0.53467400 \\
$\mathrm{O}$ & 0.080428 & -0.196465 & -1.24388200 \\
$\mathrm{H}$ & -1.457758 & 0.384639 & -1.39295800 \\
$\mathrm{O}$ & 0.293855 & -0.756527 & 1.10282900 \\
$\mathrm{H}$ & -0.499761 & -0.182314 & 1.27572100 \\
$\mathrm{~S}$ & -2.543052 & 1.484497 & 0.08877700 \\
$\mathrm{O}$ & -2.397569 & 0.687919 & -1.27248200 \\
$\mathrm{O}$ & -1.887339 & 0.650533 & 1.10634500 \\
$\mathrm{O}$ & -1.859088 & 2.75055 & -0.08122000 \\
$\mathrm{H}$ & -0.18245 & 2.399549 & -0.00512700 \\
$\mathrm{O}$ & -3.968668 & 1.540338 & 0.27778800 \\
$\mathrm{H}$ & -4.095913 & -0.396666 & 0.26681700 \\
$\mathrm{~N}$ & -3.775735 & -1.374629 & 0.19147900 \\
$\mathrm{H}$ & -2.755613 & -1.342149 & -0.00760700 \\
$\mathrm{C}$ & -4.427888 & -2.036371 & -0.95624000 \\
$\mathrm{H}$ & -5.495964 & -2.110787 & -0.77222100 \\
$\mathrm{H}$ & -3.992708 & -3.024684 & -1.07469200 \\
$\mathrm{H}$ & -4.235408 & -1.438154 & -1.84153000 \\
& -3.948537 & -2.067397 & 1.48372100 \\
$\mathrm{H}$ & -5.007187 & -2.141559 & 1.71615400 \\
$\mathrm{H} .506948$ & -3.056679 & 1.40292 \\
$\mathrm{H}$ & & &
\end{tabular}


Table S19: Geometry of $\mathrm{DMA}_{2} \mathrm{SA} \mathrm{MSA}_{2}$. Units are $\AA$.

\begin{tabular}{c|ccc} 
Atom & $\mathrm{X}$ & $\mathrm{Y}$ & $\mathrm{Z}$ \\
\hline $\mathrm{S}$ & 4.026215 & -0.02083 & 0.14005500 \\
$\mathrm{C}$ & 4.761201 & -1.601311 & 0.39052400 \\
$\mathrm{H}$ & 3.961624 & -2.337698 & 0.40619400 \\
$\mathrm{H}$ & 5.455998 & -1.785343 & -0.42256300 \\
$\mathrm{H}$ & 5.277626 & -1.568062 & 1.34534900 \\
$\mathrm{O}$ & 5.038949 & 0.96772 & -0.03205900 \\
$\mathrm{O}$ & 3.056465 & 0.178126 & 1.18765600 \\
$\mathrm{O}$ & 3.290735 & -0.178342 & -1.20988800 \\
$\mathrm{H}$ & 2.498795 & -0.835064 & -1.12345900 \\
$\mathrm{~N}$ & -2.936263 & -1.879587 & 0.70823800 \\
$\mathrm{H}$ & -2.236356 & -2.071448 & -0.04424000 \\
$\mathrm{C}$ & -4.298494 & -2.109808 & 0.19545700 \\
$\mathrm{H}$ & -5.017498 & -1.861705 & 0.97174900 \\
$\mathrm{H}$ & -4.401488 & -3.152925 & -0.09171400 \\
$\mathrm{H}$ & -4.442419 & -1.465707 & -0.66683800 \\
$\mathrm{C}$ & -2.57711 & -2.687342 & 1.88831500 \\
$\mathrm{H}$ & -3.254116 & -2.449859 & 2.70478600 \\
$\mathrm{H}$ & -1.55378 & -2.444345 & 2.15765200 \\
$\mathrm{H}$ & -2.655717 & -3.740946 & 1.63366300 \\
$\mathrm{~S}$ & -2.855963 & 1.425074 & -0.19443500 \\
$\mathrm{O}$ & -1.710474 & 2.318549 & -0.07116600 \\
$\mathrm{O}$ & -2.654762 & 0.39717 & -1.22426900 \\
$\mathrm{H}$ & -1.061543 & 0.105373 & -1.62452400 \\
$\mathrm{O}$ & -3.281632 & 0.838305 & 1.07127500 \\
$\mathrm{H}$ & 0.054977 & -1.397244 & -0.65944000 \\
$\mathrm{H}$ & -0.110715 & -0.188167 & -1.65780000 \\
$\mathrm{O}$ & -0.187654 & -0.86503 & 0.66997900 \\
$\mathrm{O}$ & -0.913701 & -2.413879 & -1.02753700 \\
$\mathrm{H}$ & -2.851014 & -0.869768 & 0.93128100 \\
$\mathrm{O}$ & 1.432831 & -1.816989 & -0.88474100 \\
$\mathrm{H}$ & 1.208381 & 0.801167 & 0.59962200 \\
$\mathrm{~N}$ & 0.866804 & 1.765438 & 0.54257100 \\
$\mathrm{H}$ & -0.124509 & 1.735888 & 0.23691400 \\
$\mathrm{C}$ & 1.655018 & 2.528317 & -0.44594700 \\
$\mathrm{H}$ & 2.692655 & 2.562842 & -0.12309400 \\
$\mathrm{H}$ & 1.240127 & 3.530596 & -0.51222500 \\
$\mathrm{H}$ & 1.581194 & 2.024922 & -1.40496300 \\
$\mathrm{H}$ & 1.9346545 & 2.334 & 1.90394300 \\
$\mathrm{H}$ & 0.289349 & 1.712827 & 2.54705400 \\
$\mathrm{H}$ & -4.195047 & 2.429365 & -0.75774200 \\
$\mathrm{H}$ & -3.36201 & 3.194853 & -0.00944400 \\
$\mathrm{H}$ & 2.873439 & -1.70540600 \\
$\mathrm{H}$ & 1.793749 & -0.878027
\end{tabular}


Table S20: Geometry of $\mathrm{DMA}_{3} \mathrm{SA}_{2} \mathrm{MSA}$. Units are $\AA$.

\begin{tabular}{c|ccc} 
Atom & $\mathrm{X}$ & $\mathrm{Y}$ & $\mathrm{Z}$ \\
\hline $\mathrm{S}$ & 4.256833 & 0.012986 & 0.01001500 \\
$\mathrm{C}$ & 5.785718 & 0.314961 & 0.84473200 \\
$\mathrm{H}$ & 6.067355 & -0.594149 & 1.36687400 \\
$\mathrm{H}$ & 6.52742 & 0.573385 & 0.09503700 \\
$\mathrm{H}$ & 5.641231 & 1.132312 & 1.54429700 \\
$\mathrm{O}$ & 3.270261 & -0.300076 & 1.05470300 \\
$\mathrm{O}$ & 4.476925 & -1.097392 & -0.89211700 \\
$\mathrm{O}$ & 3.917677 & 1.270761 & -0.66586100 \\
$\mathrm{~N}$ & 1.788212 & -2.319323 & -0.10400200 \\
$\mathrm{H}$ & 2.37113 & -1.698108 & 0.49230200 \\
$\mathrm{C}$ & 1.372418 & -1.529642 & -1.28812900 \\
$\mathrm{H}$ & 0.630606 & -2.100825 & -1.83953200 \\
$\mathrm{H}$ & 2.254416 & -1.320291 & -1.88511000 \\
$\mathrm{H}$ & 0.924928 & -0.604979 & -0.93330800 \\
$\mathrm{C}$ & 2.525881 & -3.54827 & -0.46529500 \\
$\mathrm{H}$ & 1.877894 & -4.166372 & -1.08081300 \\
$\mathrm{H}$ & 2.788416 & -4.081569 & 0.44317200 \\
$\mathrm{H}$ & 3.422745 & -3.256838 & -1.00297500 \\
$\mathrm{~S}$ & -1.913319 & 1.938573 & -0.31785000 \\
$\mathrm{O}$ & -3.176694 & 1.922064 & -1.03144400 \\
$\mathrm{O}$ & -1.247963 & 3.207422 & -0.23971900 \\
$\mathrm{O}$ & -2.040114 & 1.245138 & 0.97229400 \\
$\mathrm{H}$ & -1.18203 & -0.099828 & 1.19583900 \\
$\mathrm{O}$ & -0.898093 & 1.051026 & -1.16379200 \\
$\mathrm{H}$ & -1.248854 & 0.112212 & -1.19958700
\end{tabular}


Table S21: Geometry of $\mathrm{DMA}_{3} \mathrm{SA}_{2} \mathrm{MSA}$ (contunued). Units are $\AA$.

\begin{tabular}{c|ccc} 
Atom & $\mathrm{X}$ & $\mathrm{Y}$ & $\mathrm{Z}$ \\
\hline $\mathrm{S}$ & -1.658211 & -1.980478 & 0.33549200 \\
$\mathrm{O}$ & -0.72373 & -0.987842 & 1.13804200 \\
$\mathrm{O}$ & -1.923575 & -1.316257 & -0.94932800 \\
$\mathrm{O}$ & -0.85207 & -3.169212 & 0.21999300 \\
$\mathrm{H}$ & 0.929228 & -2.580378 & 0.39795300 \\
$\mathrm{O}$ & -2.885232 & -2.129693 & 1.09451200 \\
$\mathrm{H}$ & -3.878682 & -0.823559 & 0.55783200 \\
$\mathrm{~N}$ & -4.539066 & -0.196006 & 0.05392600 \\
$\mathrm{H}$ & -3.96609 & 0.502938 & -0.46390700 \\
$\mathrm{C}$ & -5.36656 & 0.549663 & 1.02067300 \\
$\mathrm{H}$ & -5.932171 & -0.152427 & 1.62709500 \\
$\mathrm{H}$ & -6.041248 & 1.204357 & 0.47550100 \\
$\mathrm{H}$ & -4.70139 & 1.140899 & 1.64290600 \\
$\mathrm{C}$ & -5.296857 & -1.026774 & -0.90039500 \\
$\mathrm{H}$ & -5.898671 & -1.742296 & -0.34667000 \\
$\mathrm{H}$ & -4.580994 & -1.550867 & -1.52662400 \\
$\mathrm{H}$ & -5.934613 & -0.387493 & -1.50497300 \\
$\mathrm{~N}$ & 1.53713 & 2.332055 & -0.03080700 \\
$\mathrm{C}$ & 1.806264 & 3.77865 & -0.17433900 \\
$\mathrm{C}$ & 1.177938 & 1.945094 & 1.35404600 \\
$\mathrm{H}$ & 2.403696 & 1.811297 & -0.32103000 \\
$\mathrm{H}$ & 0.299307 & 2.509249 & 1.65190700 \\
$\mathrm{H}$ & 2.028562 & 2.157003 & 1.99549400 \\
$\mathrm{H}$ & 0.978437 & 0.878514 & 1.37412500 \\
$\mathrm{H}$ & 0.911731 & 4.32527 & 0.10598800 \\
$\mathrm{H}$ & 2.065936 & 3.9857 & -1.20789900 \\
$\mathrm{H}$ & 2.643016 & 4.033488 & 0.46997500 \\
$\mathrm{H}$ & 0.761953 & 2.069382 & -0.646246 \\
$\mathrm{H}$ & 2.028562 & 2.157003 & 1.99549400 \\
$\mathrm{H}$ & 0.978437 & 0.878514 & 1.37412500 \\
$\mathrm{H}$ & 0.911731 & 4.32527 & 0.10598800 \\
$\mathrm{H}$ & 2.065936 & 3.9857 & -1.20789900 \\
$\mathrm{H}$ & 2.643016 & 4.033488 & 0.46997500 \\
$\mathrm{H}$ & 0.761953 & 2.069382 & -0.646246 \\
& & &
\end{tabular}

